\title{
Aneurysms of the petrous internal carotid artery: anatomy, origins, and treatment
}

\author{
James K. LiU, M.D., Oren N. GotTfried, M.D., Amin Amini, M.D., M.S., \\ and William T. Couldwell, M.D., Ph.D. \\ Department of Neurological Surgery, University of Utah Health Sciences Center, Salt Lake City, Utah
}

\begin{abstract}
Aneurysms arising in the petrous segment of the internal carotid artery (ICA) are rare. Although the causes of petrous ICA aneurysms remain unclear, traumatic, infectious, and congenital origins have been implicated in their development. These lesions can be detected incidentally on routine neuroimaging. Patients can also present with a wide spectrum of signs and symptoms, including cranial nerve palsies, Horner syndrome, pulsatile tinnitus, epistaxis, and otorrhagia. The treatment of petrous ICA aneurysms remains challenging. Treatment options include close observation, endovascular therapies, and surgical trapping with or without revascularization. Management dilemmas exist, particularly for incidental lesions found in asymptomatic patients. The authors review the literature and discuss the anatomy of the petrous ICA as well as the pathophysiological features of aneurysms arising in this region, and they propose a management paradigm with current treatment options.
\end{abstract}

\section{KEY WoRDS - petrous internal carotid artery aneurysm • cerebrovascular bypass • balloon occlusion • endovascular therapy • stent}

Aneurysms of the petrous segment of the ICA are rare, and their true incidence is unknown. Most are considered congenital and their morphology is fusiform.,29 Many are discovered incidentally in patients who require CT scans for other reasons. ${ }^{22}$ Typically, these lesions are large at the time of diagnosis, often with intraluminal thrombus. ${ }^{50,63}$ Petrous ICA aneurysms are thought to arise because of developmental weaknesses of the arterial wall at the sites of origin of regressed embryonic arteries including the caroticotympanic, pterygoid, vidian, stapedial, or the hyoid vessel. ${ }^{36,66}$ Histological examination demonstrates degeneration of the internal elastic lamina and medial aplasia as seen in intracranial berry aneurysms..$^{27,31}$ The management of these lesions remains challenging, particularly in those that present incidentally in asymptomatic patients. Current treatment options include close observation, proximal occlusion or trapping with endovascular balloon occlusion, coil embolization, stent placement with or without coil embolization, and surgical trapping followed by revascularization through a high-flow bypass. In this review we discuss the anatomy, pathophysiology, presentation, treatment options, and management for petrous ICA aneurysms.

\section{ANATOMY}

Although various nomenclature classification systems defining the segments of the ICA exist in the literature, we

Abbreviations used in this paper: $\mathrm{CT}=$ computerized tomography; ECA = external carotid artery; ICA = internal carotid artery; OphA $=$ ophthalmic artery. will refer to the one proposed by Bouthillier, et al., ${ }^{6}$ because it uses a numerical scale in the direction of blood flow and takes into account anatomical information and clinical considerations for neurosurgical practice. In this system, the ICA is divided into seven segments according to their adjacent anatomical structures and the compartments they traverse: $\mathrm{C} 1$, cervical; $\mathrm{C} 2$, petrous; $\mathrm{C} 3$, lacerum; C4, cavernous; C5, clinoidal; C6, ophthalmic; and C7, communicating (Fig. 1).

The cervical ICA becomes the petrous segment of the artery as it enters the petrous temporal bone at the base of the skull anterior to the internal jugular vein and medial to the styloid process (Fig. 2). The carotid canal is lined by periosteum and contains a venous plexus, postganglionic sympathetic nerves, and areolar tissue that surround the petrous ICA. The petrous ICA can be further divided into the vertical segment, the genu, and the horizontal segment. The vertical segment is approximately $10 \mathrm{~mm}$ long and turns anteromedially at the genu into the horizontal segment. The horizontal segment is approximately $20 \mathrm{~mm}$ long and courses anteromedially toward the petrous apex (Fig. 2) ${ }^{51}$ The petrous segment ends at the posterior lip of the foramen lacerum, a bone defect covered by thick fibrous or cartilaginous tissue. The ICA passes over but not through the foramen lacerum.

The petrous ICA may give off two small branches, the vidian and the caroticotympanic arteries. These arteries are small and inconstant, but may provide collateral blood flow from the ECA if ICA occlusion is present. The vidian artery (artery of the pterygoid canal) usually arises from the ECA, but may arise from the horizontal petrous ICA in some cases. It passes anteroinferiorly through the 


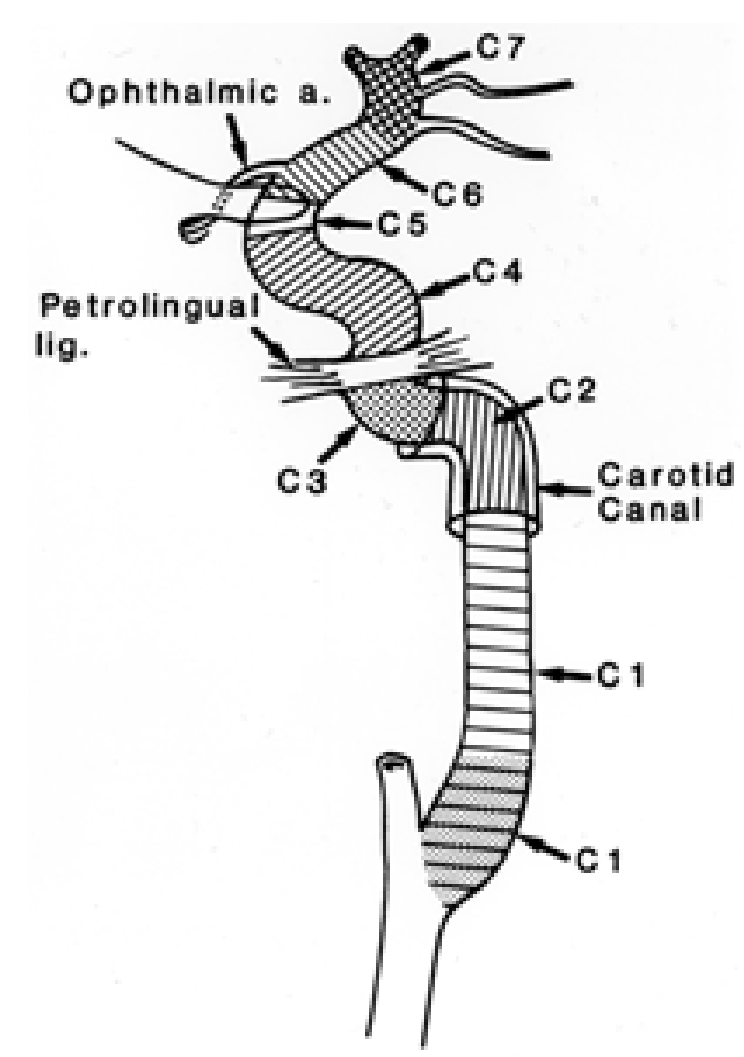

Fig. 1. Lateral anatomical diagram depicting the seven ICA segments. $\mathrm{C} 1$ = cervical segment (the bulb is indicated by stippling, and the ascending segment by horizontal lines); $\mathrm{C} 2=$ petrous segment; C3 = lacerum segment; $\mathrm{C} 4$ = cavernous segment; $\mathrm{C} 5=$ clinoidal segment; $\mathrm{C} 6=$ ophthalmic segment; $\mathrm{C} 7$ = communicating segment. a. = artery; lig. = ligament. (Reprinted with permission from Osborn AG: Diagnostic Cerebral Angiography, ed 2, Lippincott Williams \& Wilkins, Philadelphia, 1999, p 58.)

foramen lacerum and anastomoses with branches from the ECA. The caroticotympanic artery, a vestige of the embryonic hyoid artery, is a small branch that arises near the genu of the petrous ICA and passes superiorly through the stapes to supply the middle ear cavity.

Knowledge of the anatomical relationship of the petrous ICA and its neighboring neuro-otological structures is very important. The greater and lesser superficial petrosal nerves lie lateral and superior to the horizontal segment of the petrous ICA. Deeper within the temporal bone are the tensor tympani muscle and eustachian tube, which are also lateral to the horizontal petrous ICA. The cochlea is situated posterior to the genu of the petrous ICA. As this vessel angles anteromedially it is limited superiorly by the cochlea, the tympanic cavity, and the geniculate ganglion, and laterally by the greater superficial petrosal nerve. The inferior petrosal sinus lies medially and slightly posteriorly to the petrous ICA. The ICA then exits the carotid canal above the foramen lacerum at the petrous apex (Fig. 2).

\section{PATHOPHYSIOLOGY}

The origin of petrous ICA aneurysms remains unclear, although several sources have been proposed, in- cluding traumatic, mycotic, and congenital. Atherosclerosis, which plays an important role in the rise of dolichoectatic and fusiform aneurysms, tends to spare the petrous ICA. ${ }^{52}$ Lesions in the petrous ICA can be further classified as true aneurysms or pseudoaneurysms. True aneurysms are characterized by walls that are continuous with the unaffected portion of the parent vessel and can develop from a traumatically weakened arterial wall. ${ }^{24}$ Pseudoaneurysms lack a true wall and develop when a thrombus and fibrous tissue capsule forms in response to injury to all layers of an arterial wall. Such injury may result from several mechanisms, including blunt or penetrating trauma, infections, inflammation, or radiation..$^{33}$ Pseudoaneurysms form as cavities within adjacent blood clots and communicate with the lumen of the parent vessel. The lack of structural integrity of the wall of a pseudoaneurysm may result in rapid expansion if continued exposure to turbulent flow in the parent artery occurs. ${ }^{24,33}$

Trauma is a significant cause of petrous ICA aneurysms and has been well documented. ${ }^{15}$ The anatomical arrangement of an immobilized petrous ICA segment distal to a mobile cervical ICA predisposes the petrous ICA to stretch forces and traumatic injuries that may induce formation of aneurysms. ${ }^{33}$ Patients may also present with a petrous aneurysm as a result of closed head trauma with or without associated fractures into the carotid canal; these are generally pseudoaneurysms resulting from dissection of the ICA. ${ }^{2,52}$ Iatrogenic injury to the petrous ICA during myringotomy and temporal bone surgeries has been reported. . $^{1,5,7,8,12}$

Petrous ICA aneurysms of mycotic origin probably arise secondary to adventitial infection of the artery because of its close proximity to the middle ear and eustachian tube, rather than from hematogenous seeding. ${ }^{44}$ Infectious processes, including chronic otomastoiditis, tonsillar and pharyngeal infections, and cholesteatomas have been implicated in the development of these aneurysms. ${ }^{11,12,18,47,55}$ Chronic infection and the natural progressive enlargement of the aneurysm itself result in osseous erosion, rendering the ICA more susceptible to trauma or aneurysm dilation.

In the majority of cases, there is often no obvious cause and such lesions may be considered congenital in origin. ${ }^{58}$ In such cases, it has been postulated that aneurysms of the petrous ICA may arise directly from the main artery or from petrous ICA branches such as the caroticotympanic or vidian artery, the periosteal artery, and the persistent stapedial arteries. ${ }^{60,61}$ These aneurysms are thought to form as a result of developmental defects in the muscular, middle layer of the vessel. There are some arguments for a congenital origin because petrous ICA aneurysms have been diagnosed in children. ${ }^{26,35}$ There is one report of a petrous ICA aneurysm associated with von Recklinghausen neurofibromatosis. ${ }^{22}$

\section{PRESENTATION}

Petrous ICA aneurysms are usually asymptomatic. Nevertheless, they can produce a wide range of clinical signs and symptoms, depending on the aneurysm's size, direction of growth, and location within the carotid canal. Typically, patients present with symptoms in the third de- 


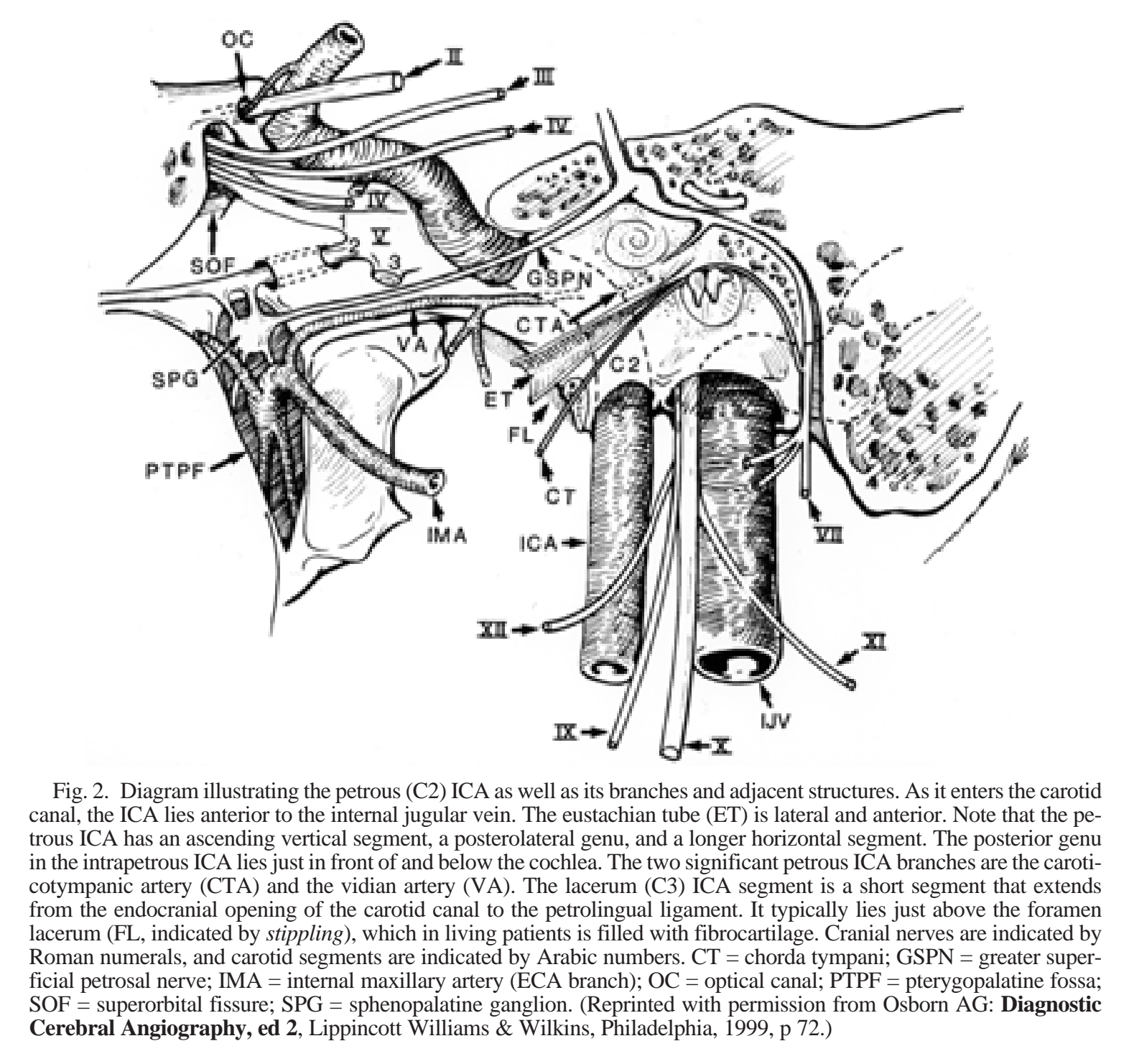

cade of life $; 9,52$ these commonly include headaches, ${ }^{10,27,52,66}$ diplopia, ${ }^{27}$ Horner syndrome, ${ }^{10,25,52}$ facial numbness, ${ }^{23,42}$ facial palsy, ${ }^{27,42}$ pulsatile tinnitus, ${ }^{27,53,66}$ dizziness ${ }^{42}$ or hearing loss. ${ }^{10,25,27,42,43,53}$ Symptoms arise as a result of hemorrhage into or mass effect on neighboring structures of the carotid canal. Various combinations of single or multiple cranial nerve deficits associated with epistaxis, otorrhagia, vertigo, and dizziness have been reported. ${ }^{25,53}$ Because the ICA is surrounded by the sympathetic plexus, aneurysms in this location can present with Horner syndrome. ${ }^{52} \mathrm{Neu}-$ rological deficits may occur due to compressive cranial nerve palsy or thromboembolic ischemic events. ${ }^{44}$

Lateral extension of the lesion into the middle ear cavity may lead to pulsatile tinnitus, progressive hearing loss, and vertigo. ${ }^{26,36,43}$ Patients who present with aural symptoms frequently present initially to an otolaryngologist, and a vascular retrotympanic mass can be detected in most cases. These often can be mistaken for a glomus tympanicum tumor, and biopsy sampling of these lesions can produce a massive hemorrhage. ${ }^{43} \mathrm{~A}$ report exists of one rare case of a patient who had a giant petrous ICA aneurysm that presented as a cerebellopontine angle mass as a result of medial erosion of the petrous bone. ${ }^{42}$ When these aneurysms spontaneously rupture, dramatic hemorrhage into the eustachian tube or middle ear can present as massive epistaxis ${ }^{9,12,46,66}$ and/or otorrhagia. ., $12,21,24,32$ This presentation is thought to occur in $25 \%$ of patients with petrous aneurysms, but the true incidence is not known. ${ }^{12}$ Such bleeding episodes can be self limited or dramatic, sometimes requiring blood transfusions to reverse hypotension and anemia. ${ }^{8,9,12,18,32}$ There have even been cases of death from exsanguination. ${ }^{2,8}$ Overall, these hemorrhages are always extracranial, and there have been no reported cases of intracranial hemorrhage from a ruptured petrous ICA aneurysm. Unlike most other intracranial aneurysms, petrous ICA lesions likely do not present with subarachnoid hemorrhage because of their extradural location.

\section{TREATMENT}

Once the diagnosis of a petrous segment ICA aneurysm is confirmed using cerebral angiography, it is important to decide if there is an indication for intervention or treatment. Patients with ruptured petrous ICA aneurysms have 
traditionally undergone treatment to prevent future bleeding or to stop an active hemorrhage. In particular, the treatment for patients with pseudoaneurysms tends to be more aggressive because of the higher risk of hemorrhage and thromboembolism.

Typically, patients with unruptured petrous ICA aneurysms who experience unremitting symptoms including cranial nerve palsies have undergone procedures to attain symptomatic relief. In contrast, there have been cases in which patients had only mild symptoms that resolved without intervention. ${ }^{23,52}$ It is difficult to determine the best management of these lesions in an asymptomatic patient or in one with only mild symptoms. In these cases, it is relevant to compare the risk associated with a proposed treatment with that related to the natural history of the disease. It is necessary to take into consideration the age of the patient, preexisting medical comorbidities, risk of hemorrhage, likelihood of morbidity and death posthemorrhage, risk of a thromboembolic event, and the risk of aneurysm growth and the resulting neurological compromise from mass effect on neighboring vascular and neural anatomy. ${ }^{23,29}$

Currently, treatment options for symptomatic and asymptomatic petrous ICA aneurysms include conservative management with serial imaging, endovascular ICA balloon occlusion, endovascular coil placement or stentassisted coil insertion, placement of a flexible covered stent, or surgical trapping and revascularization with a high-flow bypass. The treatment should be tailored to the individual, depending on the condition of the patient and the nature of the lesion. The fusiform shape of these aneurysms has made it difficult or in many cases impossible to isolate them from the carotid circulation. Therefore, early treatments were aimed at occlusion of the parent vessel, which was achieved using a number of techniques. ${ }^{10} \mathrm{Al}-$ though open surgical ligation of the common carotid artery or ICA is a well-described treatment for these aneurysms, ${ }^{32,43,52,55}$ a similar result can be achieved by a less invasive endovascular occlusion. . $^{10,14,21,25,53}$

\section{Balloon Occlusion Test and Endovascular Therapies}

Aneurysms in the petrous segment have been treated with parent artery occlusion by using endovascular balloon embolization since it was first described in $1978,{ }^{10,14}$, $21,25,53$ and more recently with coil occlusion. This method has been successful in relieving the symptoms from petrous ICA aneurysms. ${ }^{10,21,25,29}$ In this procedure, a balloon is directed into the ICA immediately proximal to the aneurysm through a guide catheter from the common femoral artery. ${ }^{10,25,53}$ First, a balloon occlusion test is performed before parent artery occlusion of the ICA to evaluate for adequate collateral flow. ${ }^{10,25,53}$ While the balloon remains inflated for 30 minutes during the test occlusion, the patient is awake and is monitored with serial neurological examinations to evaluate for new deficits and with angiography to assess the collateral circulation, including the vertebral arteries and contralateral ICA. ${ }^{10,25,53}$ (Other monitoring options include electroencephalography and transcranial Doppler ultrasonography.) ${ }^{10,53}$ If there are no adverse effects with the occlusion and if contralateral circulation is satisfactory, a detachable balloon is directed to the same site as in the occlusion study, inflated under direct fluoroscopic visualization, and detached. Then a second balloon is released proximally as a safeguard against deflation of the first balloon. ${ }^{10,25,53} \mathrm{~A}$ final injection of contrast material through the guide catheter is used to confirm complete occlusion of the aneurysm and patency of the collateral circulation..$^{9,10,25}$

Others have used embolization coils instead of the second balloon. ${ }^{12}$ Also, it is possible to perform an ICA occlusion exclusively with coil embolization. ${ }^{9}$ Nevertheless, there is a risk of coil dehiscence out of the vessel into the ear canal, which can cause life-threatening hemorrhages, especially in patients with ruptured aneurysms. ${ }^{9}$ Acute iatrogenic pseudoaneurysms located at the mesotympanum typically have no bone to encase the artery or pseudoaneurysm, thus precluding the option of coil embolization. ${ }^{1}$ Alternatively, balloon trapping procedures (balloons deposited on either side of the aneurysm) can be performed and may result in a decreased incidence of thromboembolic complications compared with proximal balloon occlusion. ${ }^{4,29}$ Nevertheless, the risk of dislodging a clot while traversing a partially thrombosed aneurysm with a detachable balloon must be strongly considered.

The major risk associated with ICA occlusion is ipsilateral cerebral infarction as a result of hypoperfusion, even in patients who have tolerated the test occlusion. ${ }^{10}$ There have been attempts to identify patients with borderline collateral flow by inducing hypotension during the test occlusion, by measuring ICA stump pressures, or by using various measures of regional cerebral perfusion. ${ }^{10}$ The risk of immediate complications from a balloon occlusion test ranges from 2 to $7 \% .{ }^{39,49,59}$ Interestingly, the incidence of ischemic complications or cerebral infarctions after ICA occlusion, even with the use of a balloon occlusion test, ranges from 2 to $22 \%{ }^{16,17,30,38,49}$ Therefore, the risk of immediate or late ischemic complications and death in patients who tolerate the balloon occlusion test can be considerable, and there is no perfect way to predict accurately the safety of an ICA occlusion. ${ }^{39}$ Some patients may also suffer strokes years after undergoing this treatment. ${ }^{39,54}$

An ICA occlusion is not a perfect treatment and there is a low incidence $(3 \%)$ of continued filling of the aneurysm after proximal balloon occlusion. ${ }^{38}$ The aneurysm may also remain patent from filling through the distal ICA or via caroticotympanic, vidian, or periosteal branches. ${ }^{10} \mathrm{An}$ other concern with ICA occlusion is the risk of cerebral aneurysm formation or growth after the procedure. , $34,35,62^{-}$ These aneurysms may appear either in the arterial territory of the occluded vessel or remote from it, and this phenomenon is estimated to occur in 0 to $10 \%$ of cases. ${ }^{28,39,48}$ Overall, endovascular vessel sacrifice may be a less invasive procedure for petrous ICA aneurysms that are unsuitable for clip occlusion, but it is not without risks, even after a successful balloon occlusion test; therefore, other, newer treatment options should be considered.

In select cases it is possible to perform a balloon or coil embolization of a petrous ICA aneurysm and to preserve the parent vessel, but because most are giant fusiform aneurysms with no definable neck, this approach is not always feasible. ${ }^{29,63,66}$ There are increasing reports of petrous ICA aneurysms being successfully treated with Guglielmi detachable coils with or without the use of a stent to preserve the parent artery, to reconstruct the incompetent arterial wall, and to prevent future complications. , $^{1,37,40,45}$ 
New, flexible intracranial stents can be used to treat ICA aneurysms in difficult-to-access areas such as the petrous segment. The stent disrupts the aneurysm inflow tract, thereby inducing stasis and facilitating intraaneurysmal thrombosis..$^{9,4}$ Also, the stent acts as a scaffold to prevent coil herniation into the parent artery, which allows tight packing of wide-necked and irregularly shaped aneurysms. ${ }^{45}$ The stent also serves as a matrix for endothelial growth.

Among several limitations of stent-assisted coil insertion is stent-induced intimal hyperplasia, which can result in hemodynamically significant stenosis. ${ }^{65}$ The high porosity of intravascular stents is another limitation for aneurysm thrombosis after stent placement. ${ }^{37}$ The novel use of covered stents allows for immediate reconstruction of the arterial wall while preserving the flow of the parent artery. ${ }^{1}$ This technology may be applied to cases of artery laceration or pseudoaneurysms of the petrous ICA. Although endovascular stent treatment with or without coil embolization of petrous ICA aneurysms appears promising, close follow up will be necessary to assess long-term patency rates and to detect delayed complications from thromboembolic events, stent-induced stenosis, or thrombus. Overall, the use of stents is a work in progress, and with new technology its application in the treatment of petrous ICA aneurysms has increased.

\section{Cerebral Revascularization With High-Flow Interpositional Bypass}

In patients with inadequate collateral flow to allow an ICA occlusion, a cerebrovascular high-flow bypass procedure can be performed, followed by endovascular aneurysm embolization, occlusion of the parent vessel, surgical trapping of the aneurysm, or resection of the lesion. ${ }^{39,44,50}$ Direct surgical exposure of petrous ICA aneurysms is a complex procedure because of the difficulties involved in accessing the lesions deep within the petrous bone. ${ }^{21}$ Early attempts at surgery were associated with high rates of mortality. ${ }^{27}$

Cerebral revascularization with a high-flow interpositional bypass is a feasible strategy in patients with complex aneurysms that cannot be directly clipped and who do not tolerate ICA occlusion, as demonstrated with a balloon occlusion test. ${ }^{10,39,53}$ As mentioned earlier, the balloon occlusion test produces false-negative results in predicting which patients will experience complications related to a surgical ICA occlusion. ${ }^{39}$ Some surgeons select revascularization procedures, even if the patient tolerates a balloon occlusion test without neurological deficits, if the individual exhibits a marked asymmetrical decrease in hemispheric cerebral blood flow $(<30 \mathrm{ml} / 100 \mathrm{~g} / \mathrm{min})$ during the study. ${ }^{57}$ For younger patients with a longer life expectancy, the goal of preserving patency of the ICA should be strongly considered.

Revascularization procedures in the hands of skilled microsurgeons yield a low morbidity and mortality rate and reduce the risk of stroke that arises with isolated ICA occlusion procedures. In a series of 61 patients requiring cerebral revascularization who were described by Lawton and coworkers, ${ }^{39} 57$ patients had good outcomes and there was only one death. In five of these patients an aneurysm was identified along the petrous segment of the ICA.
Overall, there was a $95 \%$ graft patency rate immediately postoperatively and a $92 \%$ late graft patency rate at a mean of 5.1 months after surgery. In this series, aneurysms located at the junction of the cervical and petrous ICA were resected and the parent vessel was reconstructed with a saphenous vein interposition graft. Aneurysms located in the petrous ICA were trapped proximally in the neck and distally along the petrous ICA. An entirely extradural cervical-petrous ICA bypass was performed. In another report, one patient with a petrous ICA aneurysm was successfully treated with a superficial temporal artery-middle cerebral artery bypass followed by balloon entrapment of the aneurysm without complication. ${ }^{44}$ In yet another series, ischemic symptoms were eliminated in four patients after bypass procedures for aneurysms of the petrous ICA, but these were all traumatic pseudoaneurysms. ${ }^{64}$

Several other approaches for the direct repair of petrous ICA aneurysms have been described. The first successful petrous ICA resection and primary anastomosis was reported by Glasscock and colleagues in $1983 .{ }^{24}$ It was performed via a modified infratemporal fossa approach, based on the Fisch approach for surgically removing extensive glomus jugulare tumors. ${ }^{19,20}$ After proximal and distal occlusion of the aneurysm, the intervening affected portion of the artery was removed and a primary end-toend anastomosis was performed. ${ }^{24}$ This approach is quite invasive because it involves extensive petrous bone drilling, facial nerve transposition, mandibular condylectomy, and transection and closure of the external ear canal. Alternatively, Sekhar, et al., ${ }^{56}$ advocated a combined subtemporal and preauricular infratemporal fossa approach, which affords excellent access to this region while preserving hearing and facial nerve function. Contraindications to direct repair of a petrous ICA aneurysm include poorly controlled active bleeding that requires immediate ICA occlusion or aneurysmal extension past the carotid canal, which precludes adequate exposure. ${ }^{24}$

\section{Submandibular Cervical-Supraclinoid ICA Saphenous Vein Bypass}

At our institution, we prefer the submandibular cervical-supraclinoid ICA interpositional saphenous vein graft bypass (Fig. 3). The details of this method have been previously described by the senior author (W.T.C.). ${ }^{13,41}$ This approach is less invasive than the subtemporal and infratemporal fossa approaches and does not require direct exposure of the aneurysm, which involves extensive petrous temporal bone drilling. We also prefer to tunnel the graft via the submandibular route. One advantage of this method is that it permits a more direct routing of the bypass graft to the recipient ophthalmic segment of the ICA. When the graft is tunneled superiorly and then cut to the proper length, its overall length is shortened, thereby promoting graft patency. In addition, the submandibular placement of the graft provides physical protection by the mandible, temporal muscle, and zygoma, in contrast to pre- or postauricular tunneling of the graft.

For this approach, a standard pterional craniotomy is performed on the side of the lesion. The lateral aspect of the sphenoid wing and the anterior clinoid process are removed extradurally by using a high-speed drill. This al- 


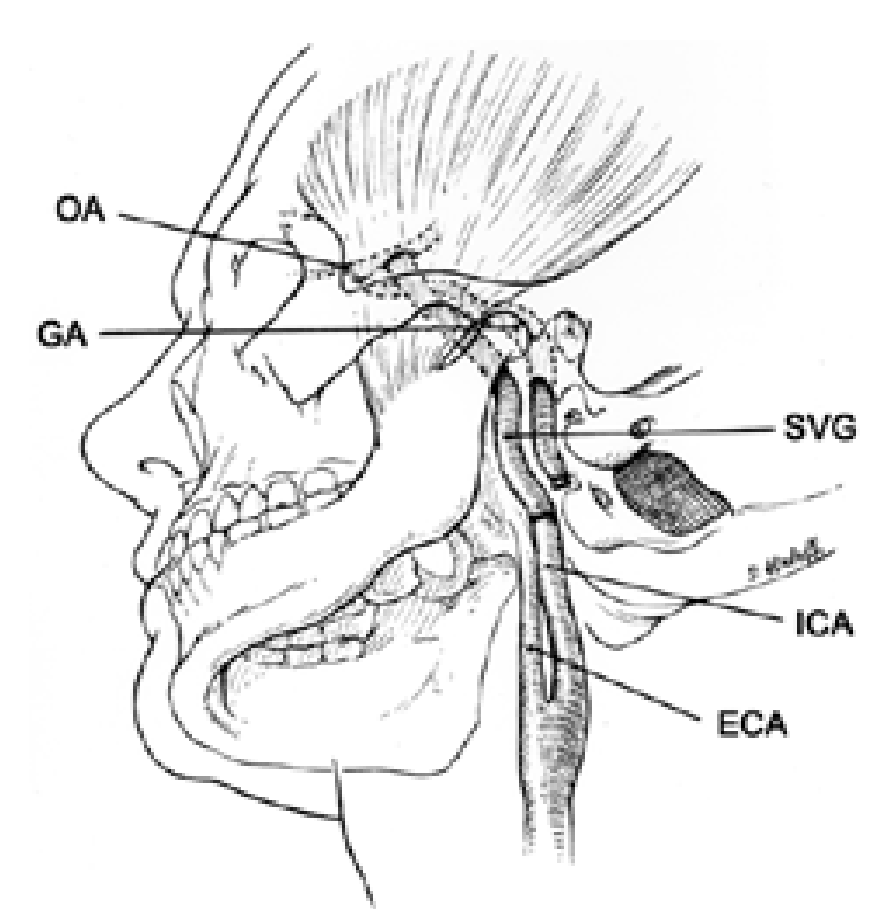

Fig. 3. Drawing showing a cervical-supraclinoid submandibular saphenous vein bypass. GA = giant petrous aneurysm (excluded); OA = OphA; SVG = saphenous vein graft. (Reprinted with permission from Couldwell WT, et al: J Neurosurg 94: 806-810, 2001.)

lows adequate exposure to the ophthalmic segment of the ICA, which serves as the recipient vessel for the saphenous vein bypass. A neck incision is made along the anterior border of the sternocleidomastoid muscle and the cervical ICA is isolated. A segment of the greater saphenous vein measuring approximately $20 \mathrm{~cm}$ is harvested and perfused with heparinized saline.

Prior to making the submandibular pass, the zygoma is removed and reflected inferiorly with the masseter and temporalis muscles. A bone trough is also made at the middle fossa skull base to provide room for the graft. This step is important in avoiding graft compromise by mandibular movement. After induction of burst suppression with intravenous propofol and administration of $5000 \mathrm{U}$ intravenous heparin, the cervical ICA is ligated as high in the neck as possible (this will ultimately shorten the graft length). An end-to-end proximal anastomosis is performed using the saphenous vein graft. The ICA may be temporarily mobilized inferiorly, which enables an easier technical proximal anastomosis. By using a short ventriculoperitoneal shunt passer or large cannula to accept the graft, the distal end is advanced beneath the mandible and through the skull base atraumatically. The graft is sized appropriately and anastomosed to the ophthalmic segment of the ICA between the OphA and posterior communicating artery branches. This is usually performed end-to-side to preserve the ophthalmic branch, or, if the OphA is not preserved, the anastomosis is performed end-to-end. A permanent vascular clip is placed, or suture ligation is performed just proximal to the OphA branch. This particular bypass technique has proved to be useful, with excellent long-term patency rates, and is an effective alternative route for interpositional vein graft placement in cases in which high-flow revascularization is desired (a case is illustrated in Figs. 4-7).

\section{MANAGEMENT PARADIGM}

Guidelines for the management of aneurysms of the petrous ICA are not well defined. There are three different situations that warrant discussion, as follows: 1) the asymptomatic patient or one with nondebilitating or mild symptoms; 2) the patient with significant symptoms including cranial nerve palsies or other neurological deficits related to mass effect from the aneurysm; and 3) the patient with a ruptured aneurysm.

\section{Asymptomatic Patients}

When deciding how to treat an asymptomatic patient, it is important to consider what is known about the natural history of petrous ICA aneurysms. Although up to $25 \%$ of patients may present with a hemorrhage, typically these are not subarachnoid in nature, patients come to medical attention in a timely manner, and most do not suffer lifethreatening bleeding episodes. Although large fusiform aneurysms sometimes have associated thrombi, there have been no reports of thromboembolic strokes as a result of these lesions. It is reasonable to recommend conservative management with serial imaging for these incidental aneurysms. If, however, the aneurysm enlarges on follow-up imaging (with or without increased symptoms from mass effect), treatment should be considered because of radiographic progression.

\section{Symptomatic Patients}

For a patient who is symptomatic from cranial neuropathies and mass effect, we recommend treatment. The options include endovascular therapy or a high-flow bypass, as discussed earlier, given that the patient is medically sta-
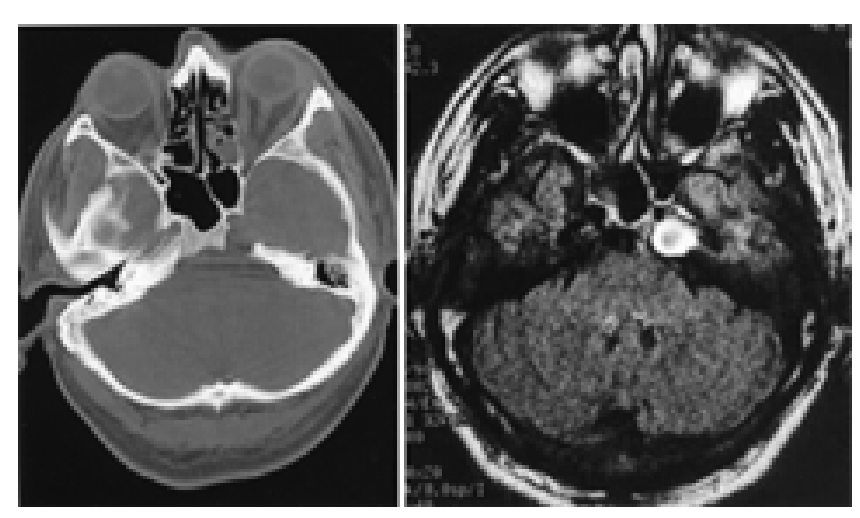

Fig. 4. Left: Preoperative CT scan with bone windows obtained in a 47-year-old man who presented with a left sixth cranial nerve palsy. The CT scan demonstrates erosion of the petrous apex and adjacent clivus on the left side. Right: Preoperative magnetic resonance image (fast low-angle inversion-recovery study) demonstrating high signal intensity within the giant petrous ICA aneurysm, with likely impingement of the abducent nerve near the region of the Dorello canal. (Reprinted with permission from Couldwell WT, et al: J Neurosurg 94:806-810, 2001.) 


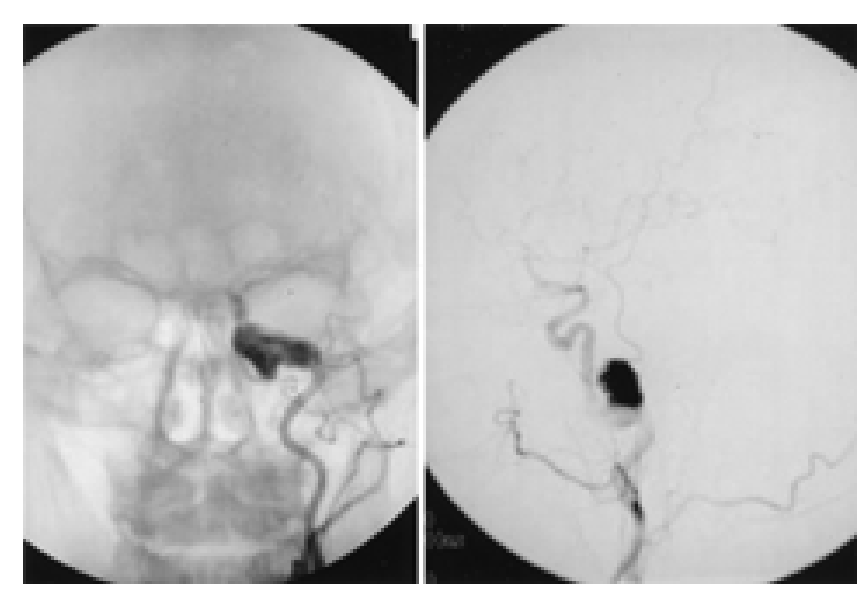

Fig. 5. Preoperative anteroposterior (left) and lateral (right) left common carotid artery angiograms demonstrating a giant petrous ICA aneurysm. (Reprinted with permission from Couldwell WT, et al: J Neurosurg 94:806-810, 2001.)

ble and able to tolerate these treatments. Endovascular therapies with balloon occlusion should be considered first if the patient is able to tolerate balloon occlusion testing. The patient should be informed of the risks related to ICA occlusion, however, especially a young patient with a long life expectancy. Endovascular stent placement with or without coil occlusion is an evolving therapy and may provide immediate reconstruction of the ICA wall while preserving the parent artery. A high-flow bypass should be considered if endovascular therapies are not feasible.

\section{Patients With Ruptured Aneurysms}

Finally, we recommend urgent treatment for a patient with a ruptured petrous ICA aneurysm. ${ }^{21,67}$ In the acute setting of an active hemorrhage, immediate endovascular balloon occlusion may be used to stabilize the patient. The use of coil embolization is not recommended by some au-

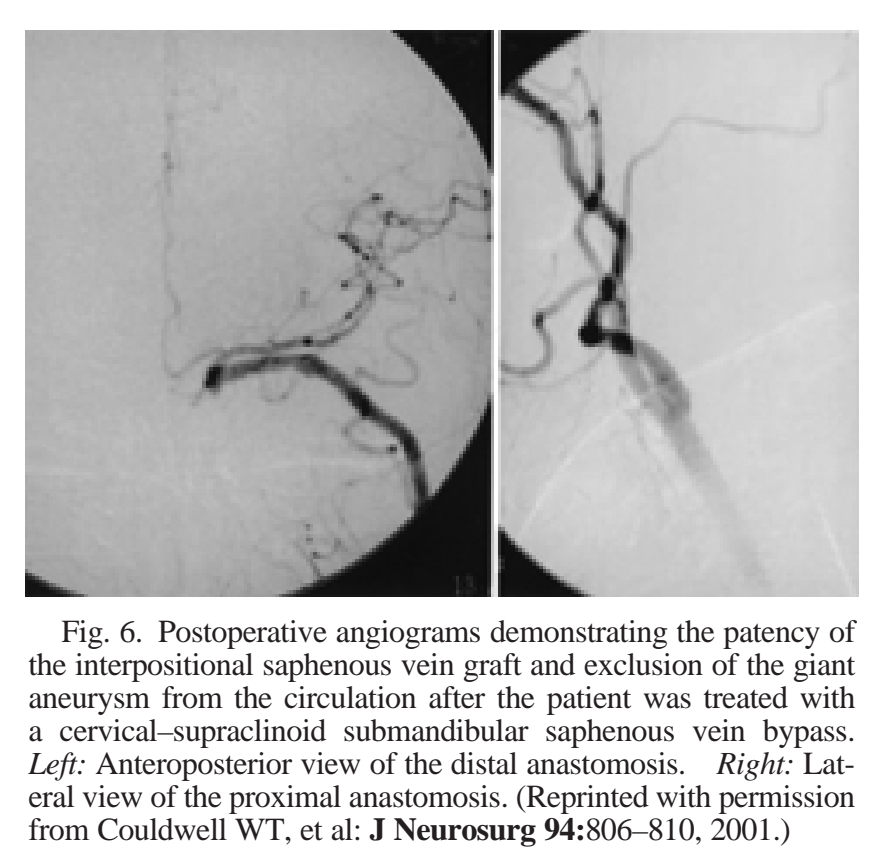

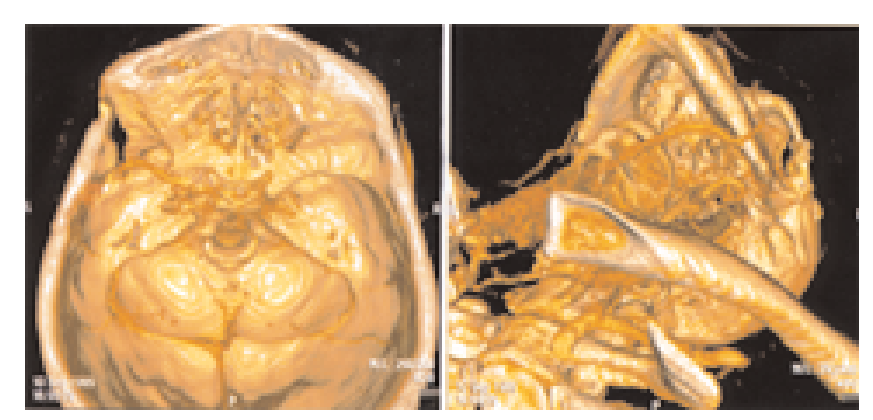

Fig. 7. Follow-up CT angiograms obtained 7 weeks postoperatively demonstrating routing of the graft and patency during mandibular movement. Left: Axial view demonstrating the graft entrance medial to the zygomatic arch, and anastomosis with the ophthalmic segment of the ICA. Note the lateral middle fossa bone removal to avoid graft compromise beneath the temporal muscle. Right: Open-mouth view demonstrating the route and patency of the saphenous interpositional graft. (Reprinted with permission from Couldwell WT, et al: J Neurosurg 94:806-810, 2001.)

thors because of the risk of coil extrusion into the ear canal in aneurysms presenting at this location. ${ }^{9}$ A high-flow bypass may be performed if necessary. ${ }^{41}$

\section{CONCLUSIONS}

The treatment of petrous ICA aneurysms remains a challenge. Management dilemmas exist when incidental lesions are found in asymptomatic patients. Treatment should be tailored to the individual and patient selection is critical. Endovascular strategies appear to be the first-line therapy if balloon occlusion testing is tolerated by the patient. Novel endovascular stent technologies, including stent-assisted coil placement and covered stents, may eliminate the aneurysm while preserving flow in the parent artery. Surgical revascularization with an interpositional high-flow bypass graft is an important strategy for lesions that are not amenable to endovascular therapy.

\section{References}

1. Alexander MJ, Smith TP, Tucci DL: Treatment of an iatrogenic petrous carotid artery pseudoaneurysm with a Symbiot covered stent: technical case report. Neurosurgery 50:658-662, 2002

2. Anderson RD, Liebeskind A, Schechter MM, et al: Aneurysms of the internal carotid artery in the carotid canal of the petrous temporal bone. Radiology 102:639-642, 1972

3. Batjer H, Mickey B, Samson D: Enlargement and rupture of distal basilar artery aneurysm after iatrogenic carotid occlusion. Neurosurgery 20:624-628, 1987

4. Berenstein A, Ransohoff J, Kupersmith M, et al: Transvascular treatment of giant aneurysms of the cavernous carotid and vertebral arteries. Functional investigation and embolization. Surg Neurol 21:3-12, 1984

5. Botma M, Kell RA, Bhattacharya J, et al: Aberrant internal carotid artery in the middle-ear space. J Laryngol Otol 114: 784-787, 2000

6. Bouthillier A, van Loveren HR, Keller JT: Segments of the internal carotid artery: a new classification. Neurosurgery 38: 425-433, 1996

7. Brodish BN, Woolley AL: Major vascular injuries in children undergoing myringotomy for tube placement. Am J Otolaryngol 20:46-50, 1999

8. Busby DR, Slemmons DH, Miller TF Jr: Fatal epistaxis via ca- 
rotid aneurysm and eustachian tube. Arch Otolaryngol 87: 295-298, 1968

9. Cheng KM, Chan CM, Cheung YL, et al: Endovascular treatment of radiation-induced petrous internal carotid artery aneurysm presenting with acute haemorrhage. A report of two cases. Acta Neurochir 143:351-356, 2001

10. Coley SC, Clifton A, Britton J: Giant aneurysm of the petrous internal carotid artery: diagnosis and treatment. J Laryngol Otol 112:196-198, 1998

11. Cook MR, Fernandes CM: Mycotic aneurysms of the petrous portion of the internal carotid artery. A report of 2 cases. S Afr J Surg 21:109-115, 1983

12. Costantino PD, Russell E, Reisch D, et al: Ruptured petrous carotid aneurysm presenting with otorrhagia and epistaxis. Am J Otol 12:378-383, 1991

13. Couldwell WT, Zuback J, Onios E, et al: Giant petrous carotid aneurysm treated by submandibular carotid-saphenous vein bypass. Case report. J Neurosurg 94:806-810, 2001

14. Debrun G, Lacour P, Caron JP, et al: Detachable balloon and calibrated-leak balloon techniques in the treatment of cerebral vascular lesions. J Neurosurg 49:635-649, 1978

15. Depauw P, Defreyne L, Dewaele F, et al: Endovascular treatment of a giant petrous internal carotid artery aneurysm. Case report and review of the literature. Minim Invasive Neurosurg 46: $250-253,2003$

16. Drake CG, Peerless SJ, Ferguson GG: Hunterian proximal arterial occlusion for giant aneurysms of the carotid circulation. $\mathbf{J}$ Neurosurg 81:656-665, 1994

17. Eckert B, Thie A, Carvajal M, et al: Predicting hemodynamic ischemia by transcranial Doppler monitoring during therapeutic balloon occlusion of the internal carotid artery. AJNR 19: 577-582, 1998

18. Ehni G, Barrett JH: Hemorrhage from the ear due to an aneurysm of the internal carotid artery. N Engl J Med 262: 1323-1325, 1960

19. Fisch U: Infratemporal fossa approach for glomus tumors of the temporal bone. Ann Otol Rhinol Laryngol 91:474-479, 1982

20. Fisch U: Infratemporal fossa approach to tumors of the temporal bone and base of the skull. J Laryngol Otol 92:949-967, 1978

21. Forshaw MA, Higgins N, Hardy DG, et al: Rupture of an internal carotid artery aneurysm in the petrous temporal bone. Br J Neurosurg 14:479-482, 2000

22. Frank E, Brown BM, Wilson DF: Asymptomatic fusiform aneurysm of the petrous carotid artery in a patient with von Recklinghausen's neurofibromatosis. Surg Neurol 32:75-78, 1989

23. Gibson RD, Cowan IA: Giant aneurysm of the petrous carotid artery presenting with facial numbness. Neuroradiology 31: 440-441, 1989

24. Glassock ME III, Smith PG, Bond AG, et al: Management of aneurysms of the petrous portion of the internal carotid artery by resection and primary anastomosis. Laryngoscope 93: 1445-1453, 1983

25. Goodman TR, Renowden S, Byrne JV: Case report: petrous internal carotid artery aneurysm: an unusual cause of eustachian tube dysfunction. Clin Radiol 51:658-660, 1996

26. Guha A, Montanera W, Hoffman HJ: Congenital aneurysmal dilatation of the petrous-cavernous carotid artery and vertebral basilar junction in a child. Neurosurgery 26:322-327, 1990

27. Guirguis S, Tadros FW: An internal carotid aneurysm in the petrous temporal bone. J Neurol Neurosurg Psychiatry 24: 84-85, 1961

28. Gurdjian ES, Lindner DW, Thomas LM: Experiences with ligation of the common carotid artery for treatment of aneurysms of the internal carotid artery. J Neurosurg 23:311-318, 1965

29. Halbach VV, Higashida RT, Hieshima GB, et al: Aneurysms of the petrous portion of the internal carotid artery: results of treatment with endovascular or surgical occlusion. AJNR 11: 253-257, 1990
30. Higashida RT, Halbach VV, Dowd C, et al: Endovascular detachable balloon embolization therapy of cavernous carotid artery aneurysms: results in 87 cases. J Neurosurg 72: 857-863, 1990

31. Hiranandani LH, Chandra O, Malpani NK, et al: An internal carotid aneurysm in the petrous temporal bone. J Laryngol Otol 76:703-706, 1962

32. Holtzman RN, Parisier SC: Acute spontaneous otorrhagia resulting from a ruptured petrous carotid aneurysm. Case report. J Neurosurg 51:258-261, 1979

33. Hwang CJ, Moonis G, Hurst RW, et al: Bilateral petrous internal carotid artery pseudoaneurysms presenting with sensorineural hearing loss. AJNR 24:1139-1141, 2003

34. Jaffe ME, McHenry LC Jr: Cerebral aneurysm following spontaneous carotid occlusion. Neurology 18:1012-1014, 1968

35. Johnston SC, Halbach VV, Smith WS, et al: Rapid development of giant fusiform cerebral aneurysms in angiographically normal vessels. Neurology 50:1163-1166, 1998

36. Kudo S, Colley DP: Multiple intrapetrous aneurysms of the internal carotid artery. AJNR 4:1119-1121, 1983

37. Lanzino G, Wakhloo AK, Fessler RD, et al: Efficacy and current limitations of intravascular stents for intracranial internal carotid, vertebral, and basilar artery aneurysms. J Neurosurg 91:538-546, 1999

38. Larson JJ, Tew JM Jr, Tomsick TA, et al: Treatment of aneurysms of the internal carotid artery by intravascular balloon occlusion: long-term follow-up of 58 patients. Neurosurgery 36: 26-30, 1995

39. Lawton MT, Hamilton MG, Morcos JJ, et al: Revascularization and aneurysm surgery: current techniques, indications, and outcome. Neurosurgery 38:83-94, 1996

40. Lempert TE, Halbach VV, Higashida RT, et al: Endovascular treatment of pseudoaneurysms with electrolytically detachable coils. AJNR 19:907-911, 1998

41. Liu JK, Couldwell WT: Interpositional carotid artery bypass strategies in the surgical management of aneurysms and tumors of the skull base. Neurosurg Focus 14 (3):Article 2, 2003

42. Love MH, Bell KE: Case report: giant aneurysm of the intrapetrous carotid artery presenting as a cerebellopontine angle mass. Clin Radiol 51:587-588, 1996

43. Lynch JC, Amaral MA, Pareira A: Giant aneurysm of the petrous portion of the carotid artery. J Neurol Neurosurg Psychiatry 46:685-687, 1983

44. McGrail KM, Heros RC, Debrun G, et al: Aneurysm of the ICA petrous segment treated by balloon entrapment after EC-IC bypass. Case report. J Neurosurg 65:249-252, 1986

45. Mericle RA, Lanzino G, Wakhloo AK, et al: Stenting and secondary coiling of intracranial internal carotid artery aneurysm: technical case report. Neurosurgery 43:1229-1234, 1998

46. Moffat DA, O'Connor AF: Bilateral internal carotid aneurysms in the petrous temporal bones. Arch Otolaryngol 106: $172-175,1980$

47. Morantz RA, Kirchner FR, Kishore P: Aneurysms of the petrous portion of the internal carotid artery. Surg Neurol 6: 313-318, 1976

48. Nishioka H: Results of the treatment of intracranial aneurysms by occlusion of the carotid artery in the neck. J Neurosurg 25: 660-704, 1966

49. Origitano TC, al-Mefty O, Leonetti JP, et al: Vascular considerations and complications in cranial base surgery. Neurosurgery 35:351-363, 1994

50. Patrick JT: Magnetic resonance imaging of petrous carotid aneurysms. J Neuroimaging 6:177-179, 1996

51. Paullus WS, Pait TG, Rhoton AI Jr: Microsurgical exposure of the petrous portion of the carotid artery. J Neurosurg 47: 713-726, 1977

52. Rawlinson J, Colquhoun IR: Aneurysms involving the intrapetrous internal carotid artery: a rare cause of Horner's syndrome. Br J Radiol 63:69-72, 1990 


\section{Aneurysms of the petrous ICA: anatomy, origins, and treatment}

53. Reece PH, Higgins N, Hardy DG, et al: An aneurysm of the petrous internal carotid artery. J Laryngol Otol 113:55-57, 1999

54. Roski RA, Spetzler RF, Nulsen FE: Late complications of carotid ligation in the treatment of intracranial aneurysms. J Neurosurg 54:583-587, 1981

55. Samuel J, Fernandes CM: Mycotic aneurysm of the petrous portion of the internal carotid artery. J Laryngol Otol 103: 111-114, 1989

56. Sekhar LN, Schramm VL Jr, Jones NF, et al: Operative exposure and management of the petrous and upper cervical internal carotid artery. Neurosurgery 19:967-982, 1986

57. Sekhar LN, Sen CN, Jho HD: Saphenous vein graft bypass of the cavernous internal carotid artery. J Neurosurg 72:35-41, 1990

58. Stallings JO, McCabe BF: Congenital middle ear aneurysm of internal carotid. Arch Otolaryngol 90:39-43, 1969

59. Tarr RW, Jungreis CA, Horton JA, et al: Complications of preoperative balloon test occlusion of the internal carotid arteries: experience in 300 cases. Skull Base Surgery 1:240-244, 1991

60. Teal JS, Bergeron RT, Rumbaugh CL, et al: Aneurysms of the petrous or cavernous portions of the internal carotid artery associated with nonpenetrating head trauma. J Neurosurg 38: 568-574, 1973

61. Teal JS, Rumbaugh CL, Segall HD, et al: Anomalous branches of the internal cartoid artery. Radiology 106:567-573, 1973

62. Timperman PE, Tomsick TA, Tew JM Jr, et al: Aneurysm formation after carotid occlusion. AJNR 16:329-331, 1995
63. Urwin RW, Higashida RT, Halbach VV, et al: Endovascular therapy for the carotid artery. Neuroimaging Clin N Am 6: 957-973, 1996

64. Vishteh AG, Marciano FF, David CA, et al: Long-term graft patency rates and clinical outcomes after revascularization for symptomatic traumatic internal carotid artery dissection. Neurosurgery 43:761-768, 1998

65. Wakhloo AK, Lanzino G, Lieber BB, et al: Stents for intracranial aneurysms: the beginning of a new endovascular era? Neurosurgery 43:377-379, 1998

66. Willinsky R, Lasjaunias P, Pruvost P, et al: Petrous internal carotid aneurysm causing epistaxis: balloon embolization with preservation of the parent vessel. Neuroradiology 29:570-572, 1987

67. Winslow N: Extracranial aneurysm of the internal carotid artery: history and analysis of the cases registered up to Aug. 1, 1925. Arch Surg 13:689-729, 1926

Manuscript received September 17, 2004.

Accepted in final form October 5, 2004.

Address reprint requests to: William T. Couldwell, M.D., Ph.D., Department of Neurological Surgery, University of Utah Medical Center, 30 North 1900 East, Suite 3B409, Salt Lake City, Utah 84132. email: william.couldwell@hsc.utah.edu. 